\title{
Collagen Membranes: A Review
}

\author{
Pintippa Bunyaratavej* and Hom-Lay Wang ${ }^{\dagger}$
}

Collagen materials have been utilized in medicine and dentistry because of their proven biocompatability and capability of promoting wound healing. For guided tissue regeneration (GTR) procedures, collagen membranes have been shown to be comparable to non-absorbable membranes with regard to probing depth reduction, clinical attachment gain, and percent of bone fill. Although these membranes are absorbable, collagen membranes have been demonstrated to prevent epithelial downgrowth along the root surfaces during the early phase of wound healing. The use of grafting material in combination with collagen membranes seems to improve clinical outcomes for furcation, but not intrabony, defects when compared to the use of membranes alone. Recently, collagen materials have also been applied in guided bone regeneration (GBR) and root coverage procedures with comparable success rates to non-absorbable expanded polytetrafluoroethylene (ePTFE) membranes and conventional subepithelial connective tissue grafts, respectively. Long-term clinical trials are still needed to further evaluate the benefits of collagen membranes in periodontal and peri-implant defects. This article will review the rationale for each indication and its related literature, both in vitro and in vivo studies. The properties that make collagen membranes attractive for use in regenerative therapy will be addressed. In addition, varieties of cross-linking techniques utilized to retard the degradation rate of collagen membranes will be discussed. J Periodontol 2001; 72:215-229.

\section{KEY WORDS}

Collagen/therapeutic use; guided tissue regeneration; guided bone regeneration; membranes, barrier; membranes, bioabsorbable; tooth root; wound healing.

\footnotetext{
* Previously, Department of Periodontics/Prevention/Geriatrics, School of Dentistry, University of Michigan, Ann Arbor, MI; currently, Department of Periodontology, Faculty of Dentistry, Chiang Mai University, Chiang Mai, Thailand.

$\dagger$ Department of Periodontics/Prevention/Geriatrics, School of Dentistry, University of Michigan.
}

T raditional periodontal surgeries are often performed to gain access to diseased root surfaces and remove local factors, i.e., plaque, calculus, and endotoxin. Periodontal surgical techniques include gingivectomy, open flap debridement, modified Widman flap, and osseous surgery, which may offer probing depth reduction and gain of clinical attachment. ${ }^{1-3}$ However, new attachment achieved by these procedures is usually a result of the formation of long junctional epithelium with slight or no new connective tissue attachment and negligible new cementum formation. ${ }^{4-7}$ In contrast, guided tissue regeneration (GTR), the procedure in which a barrier is utilized to exclude epithelium from the root surfaces, ${ }^{8}$ has been shown to partially regenerate lost periodontal tissue with new bone, periodontal ligament, and cementum. ${ }^{9}$ Several materials, both nonabsorbable and bioabsorbable barriers, have been developed and modified in an attempt to achieve complete periodontal regeneration. ${ }^{9}$ More recently, the membrane barriers have also been applied for regenerating bone surrounding periimplant defects, in the so-called guided bone regeneration (GBR) procedure. ${ }^{10}$ The purpose of this article is to address the uses of collagen membranes for their roles in GTR, GBR, and other applications. The properties that make collagen membranes attractive for use in regenerative therapy will be addressed. The related literature, both in vitro and in vivo studies, as well as clinical trials will be discussed. Moreover, a description of how collagen membranes have been used clinically and the future role of this material in dental treatment will be explored. 


\section{Collagen Membranes and Their Properties}

Due to the need for a second surgery to retrieve nonabsorbable membranes, a demand for bioabsorbable membranes with comparable, if not better, clinical outcomes became apparent. Type I collagen is a predominant component found in periodontal connective tissue. Several commercially available collagen membranes have been developed using type I collagen as their major component (Table 1). In addition, collagen materials also possess additional advantages including hemostasis, chemotaxis for periodontal ligament (PDL) fibroblasts ${ }^{11,12}$ and gingival fibroblasts, ${ }^{13}$ weak immunogenicity, ${ }^{14-16}$ easy manipulation, and ability to augment tissue thickness. ${ }^{17}$ Hence, collagen material appears to be an ideal choice for an absorbable GTR barrier. It should be noted that although the main component of collagen membranes discussed in this paper is type I collagen, these membranes are, in fact, not identical depending on sources of material (bovine or porcine) and additional components. However, for the purposes of this review, these membranes are all considered as collagen membranes, while details of the components and cross-linking techniques are provided in the tables.
Quteish et al. ${ }^{18}$ evaluated the biocompatability and resorption pattern of a human collagen graft material both in vitro and in vivo. Human collagen, extracted from placenta, was implanted subcutaneously in 10 Sprague Dawley rats. The graft was encapsulated by day 7 and was slowly resorbed over 56 days with minimal inflammatory response. Locci et al. ${ }^{13}$ compared the degree of biocompatability between collagen and polytetrafluoroethylene (PTFE). Using ${ }^{3} \mathrm{H}$-thymidine, it was found that PTFE membrane inhibited gingival fibroblast DNA synthesis, while the matrix membrane, consisting of $95 \%$ collagen and 5\% chondroitin-4-sulfate, enhanced cell proliferation. In addition, the PTFE membrane caused a marked decrease in synthesis of extracellular collagen and glycosaminoglycan (GAG), the major components of extracellular matrix (ECM). Collagen matrix resulted in a significantly increased production of these components. Since ECM plays a major role in wound healing, the authors proposed that collagen may be more suitable than PTFE barrier to achieve periodontal regeneration. Schlegel et al. ${ }^{16}$ evaluated the immune responses to the collagen membrane both in vivo and in a pilot study in

\section{Table I.}

Collagen Membranes Currently Available or Under Investigation*

\begin{tabular}{|c|c|c|c|c|c|}
\hline Name & Company & Sources & Methods of Cross-Link & Main Components & Resorption Rate $^{\dagger}$ \\
\hline BioMend & $\begin{array}{l}\text { Sulzer Calcitek, } \\
\text { Carlsbad, CA }\end{array}$ & Bovine tendon & Formaldehyde & I00\% type I collagen & 6-8 weeks \\
\hline BioMend-Extend & $\begin{array}{l}\text { Sulzer Calcitek, } \\
\text { Carlsbad, CA }\end{array}$ & Bovine tendon & Formaldehyde & I00\% type I collagen & 18 weeks \\
\hline Periogen & $\begin{array}{l}\text { Collagen Inc., } \\
\text { Palo Alto, CA }\end{array}$ & Bovine dermis & Glutaraldehyde & Types I and III collagen & 4-8 weeks \\
\hline Paroguide & Coletica, Lyon, France & Calfskin & DPPA $\ddagger$ & $\begin{array}{l}96 \% \text { type I collagen } \\
\text { and } 4 \% \text { chondroitin- } \\
\text { 4-sulfate }\end{array}$ & 4-8 weeks \\
\hline Biostite & Coletica, Lyon, France & Calfskin & DPPA & $\begin{array}{l}88 \% \text { hydroxyapatite, } \\
9.5 \% \text { type I collagen, and } \\
2.5 \% \text { chondroitin- } 4- \\
\text { sulfate }\end{array}$ & 4-8 weeks \\
\hline BioGide & $\begin{array}{l}\text { Geistlich, Wolhusen, } \\
\text { Switzerland }\end{array}$ & Porcine dermis & None & Types I and III collagen & 24 weeks \\
\hline Tissue Guide & $\begin{array}{l}\text { Koken Co., Tokyo, } \\
\text { Japan }\end{array}$ & Bovine dermis + tendon & HMDIC§ & $\begin{array}{l}\text { Atelocollagen }\left(1^{\circ}\right)+ \\
\text { tendon collagen }\end{array}$ & 4-8 weeks \\
\hline BioBar & $\begin{array}{l}\text { Colbar Research \& } \\
\text { Dev. Ltd., Ramat- } \\
\text { Hasharon, Israel }\end{array}$ & Bovine tendon & N/A & I00\% type I collagen & 6-8 months \\
\hline
\end{tabular}

* Modified from reference 89.

$\dagger$ Company data.

‡ Diphenylphosphorylazide.

$\S$ Hexamethylenediisocyanate. 
humans. Using enzyme-linked immunosorbent assay (ELISA), the authors found no specific immunoreaction against collagen during an 8- to 65-day observation period. Thus, this confirmed the lack of immunogenicity of collagen membrane previously reported by Johns et al. ${ }^{19}$ and Wang et al. ${ }^{20}$

The ideal properties of a barrier for GTR procedures include the ability to exclude unwanted epithelial cells and maintain a space for appropriate cells (e.g., PDL cells, bone cells, and/or cementoblasts) to repopulate the wounded area. Thus, a membrane must maintain its structural integrity during early wound healing. The degradation rate of collagen membranes varies depending on the source. Unsal et al. ${ }^{21}$ compared different collagen materials including fascia lata, fascia temporalis, dura mater, and bovine origin. By placing the collagen materials subcutaneously in rats for up to 42 days, the authors observed that type I bovine collagen and fascia lata were resorbed faster and that the majority of materials were replaced by normal connective tissue after 42 days. In contrast, the resorption rates of dura mater and fascia temporalis were much slower. However, whether this information from an in vivo study performed subcutaneously could be applied to the human oral cavity requires further investigation.

Paul et al. ${ }^{22}$ examined the degradation rate of noncross-linked bovine collagen in an attempt to treat Class II furcation defects. No residual collagen material protruding from the gingival margin was noted at one week. However, Pitaru et al. ${ }^{23}$ reported a half-life of 21 to 28 days for non-cross-linked collagen when implanted intramuscularly or subcutaneously. These differences in degradation times between these 2 studies may be attributed to different study designs with regard to placement site; i.e., intramuscularly or subcutaneous versus intraoral, as well as a closed versus an open environment. Several periodontal pathogens (e.g., Porphyromonas gingivalis, Bacteroides melaninogenicus) have been reported to be capable of producing collagenase ${ }^{24,25}$ that may degrade membranes prematurely. In addition, it should be noted that Paul et al. $^{22}$ intentionally exposed the coronal part of the collagen membrane. This may allow bacterial colonization, resulting in early degradation and ultimately compromising the final outcomes. ${ }^{26-28}$

To prolong the absorption time of collagen membranes, various cross-linking techniques have been developed. These include ultraviolet light, ${ }^{23,29}$ hexamethylenediisocyanate (HMDIC), 29,30 glutaraldehyde plus irradiation, ${ }^{31}$ and diphenylphosphorylazide. ${ }^{32,33}$ Pitaru et al. ${ }^{23}$ treated surgically created defects in dogs with cross-linked collagen membranes obtained from rat-tail tendons. They reported partial regeneration consisting of new bone, PDL, and cementum in the apical half, with long junctional epithelium and connective tissue attachment in the coronal half of defects.
However, only long junctional epithelium with negligible new bone was observed in sites treated with flap debridement only. The authors attributed partial regeneration to early membrane exposure, resulting in quick degradation of the membrane. Using 250 rats, Minabe et al. ${ }^{29}$ studied differences in wound healing between atelocollagen (purified from bovine dermis) and insoluble collagen (purified from bovine tendon) crosslinked with ultraviolet rays or HMDIC. Two layers of membrane were placed under palatal flaps in the treatment groups, while flaps were closed without any membrane in the control group. The greatest inhibitory effect on epithelial downgrowth was observed in the HMDIC weak cross-linked atelocollagen subgroup $(31.2 \%)$. In addition, it was noted that the non-crosslinked collagen membrane disappeared in 5 to 7 days, while residues of the cross-linked collagen membrane were still observed at day 14 . The authors concluded that the higher the degree of cross-linking, the slower the rate of degradation of the collagen membrane.

Since a non-absorbable (ePTFE) membrane is required to stay in place for 4 to 6 weeks before being surgically removed, one may question the effectiveness of the collagen membrane in enhancing regeneration due to its relatively fast degradation rate. Minabe et al. ${ }^{29}$ demonstrated that rapid epithelial proliferation occurred on days 3 to 5 , and then progressed up to 14 days in the non-cross-linked collagen group. In contrast, the rate of epithelial proliferation increased slightly up to day 5 with no subsequent downgrowth in the crosslinked atelocollagen group. Similar results were also obtained by Kodama et al. ${ }^{30}$ in a 28 -day study in 120 Wistar rats. In this study, the effect of collagen concentration in HMDIC cross-linked atelocollagen was evaluated. Regardless of the concentration, collagentreated sites demonstrated a significantly slower rate of apical migration and more perpendicular fiber bundles compared to untreated controls. Thus, from these studies it may be postulated that the period of time in which a collagen membrane remains intact is sufficient for preventing apical migration of epithelium during early periodontal wound healing, since the critical time for epithelial proliferation occurs within the first 14 days. Furthermore, Numabe et al., ${ }^{34}$ using ${ }^{3} \mathrm{H}$-thymidine autoradiography, observed epithelial cell kinetics during periodontal wound healing with and without atelocollagen membrane in a rat model. The authors reported that in the collagen membrane group, ${ }^{3} \mathrm{H}$ thymidine labeled cells were noted in both basal and prickle cell layers of newly formed sulcular epithelium, compared to only a single layer of ${ }^{3} \mathrm{H}$-thymidine-incorporated basal cells along the root surface in non-membrane treated controls. In addition, there was no apical extension of the epithelium beyond the cemento-enamel junction (CEJ) in the collagen group even though the membrane was completely resorbed at day 14 . These 
findings implied that the collagen membrane decreases the mitotic activity of basal cells resulting in piling up of dividing cells adjacent to the membrane, thus inhibiting apical migration of the epithelium. Although these reports showed an adequate barrier function of the collagen materials, it is noteworthy that most studies were conducted in a rat model in a short period of time. Further studies in this area are still needed.

It should be noted that a prolonged resorption rate of the collagen membrane does not always result in greater periodontal/bone regeneration. Using rat calvarial defects, Brunel et al. ${ }^{32}$ evaluated the effect of degrees of cross-linking on guided bone regeneration. Their results confirmed previous reports that the higher degree of cross-linking, the longer the resorption rate, and more defect fills were noted on membrane-treated sites. However, no statistically significant differences in bone fills were observed among sites treated with collagen membranes with different cross-linking degrees. Interestingly, highly cross-linked, slow-resorbing collagen membranes appeared to have negative effects on final clinical outcomes as reported by Crigger et al. ${ }^{35}$ in a dog model. The authors found the greatest attachment gain when using weak cross-linked, rapid-resorbing collagen membranes, followed by ePTFE membranes and high cross-linked collagen membranes. Membrane exposure, one of the primary contributing factors for GTR/GBR failure, was commonly found in ePTFE membranes and highly cross-linked collagen membranes. At 6 months, the highly cross-linked membrane group demonstrated extensive recession and loss of attachment. In contrast, the weak cross-linked collagen showed $84 \%$ new connective tissue attachment while ePTFE membranes had only $53 \%$.

Regarding cross-linking techniques, the glutaraldehyde technique, the most widely used chemical crosslinking technique, was reported to leave cytotoxic residue during the process. ${ }^{36}$ To overcome the drawback of this technique, the diphenylphosphorylazide (DPPA) technique was developed. Collagen barriers manufactured through this process showed comparable results when compared to other collagen membranes. ${ }^{33}$

In addition to cross-linking, heparan sulfate and fibronectin have also been added in an attempt to enhance the inhibitory effect on epithelial migration. In a dog model, Pitaru et al. ${ }^{37}$ compared the length of the root surfaces repopulated by connective tissue between sites treated with collagen membrane alone, and those treated with a fibronectin and heparan sulfate-enriched collagen membrane. Approximately 30\% more root surfaces were repopulated with connective tissue in the fibronectin and heparan sulfate-treated group compared to the collagen alone group.

Overall, studies have shown that collagen materials have an ability to inhibit apical migration of epithe- lium regardless of the fast degradation rate. It should be noted that once the epithelium becomes mature, membrane integrity is no longer essential for tissue regeneration. Hence, the crucial period for the membrane to remain intact is during the early wound healing period when the epithelium actively migrates along the root surface. ${ }^{38}$ After that, the presence of a barrier may cause a delay in connective tissue maturation during the late phase of wound healing.

\section{Animal Studies}

Several animal studies have been conducted to evaluate the safety and efficacy of collagen barriers (Table 2 ). Most earlier studies focused on collagen biocompatibility and its resorption rate, ${ }^{12,13,16,29,30,34}$ and the majority of them used surgically created defects. In addition, most studies ended within 4 to 12 weeks, with few studies lasting for 6 months. These factors should be considered when applying results obtained from these in vivo reports. Nevertheless, the conclusions drawn from these animal studies can be used as a reference for the material. They are: 1) collagen membranes are weak immunogen and biocompatible; 2) collagen membranes possess the chemotactic ability to attract gingival and/or PDL fibroblasts, thus promoting proliferation and extracellular matrix maturation, and facilitating wound healing; ${ }^{13,16} 3$ ) collagen membranes have been shown to be an effective barrier in preventing apical migration of epithelium; $29,30,34$ 4 ) the higher degree of cross-linking, the slower the resorption rate;29-33,35 and 5) collagen membranes provide better clinical results when compared to open flap debridement alone. ${ }^{23,35,37,39,40}$

\section{Guided Tissue Regeneration}

Intrabony defects. Since the late 1980s, collagen membranes have been tested for their ability to promote regeneration in periodontal intrabony defects (Table 3). ${ }^{31,41-46}$ Most studies have shown significantly greater probing depth (PD) reduction, clinical attachment gain, and bone fill in collagen-treated groups than open debridement controls. ${ }^{31,42-45}$ The mean PD reduction from these studies ranged from 3.1 to 5.5 $\mathrm{mm}$ in collagen-treated groups, while only 2.2 to 2.8 $\mathrm{mm}$ reductions were noted in controls. However, Tanner et al. ${ }^{41}$ showed no difference in clinical attachment gain when comparing collagen membranes to flap debridement alone. It should be noted that this study was done in only 4 patients with hopeless teeth, and results were obtained only at 3 months. Other studies had more participants (9 to 52) with longer follow-up (6 months). Factors contributing to these variable results include different cross-linking techniques, initial probing depths, width of defects, measuring techniques, and different analytical methods. ${ }^{47}$ For example, Chung et al. ${ }^{42}$ used mean PD and attachment level from each tooth for data analysis instead 


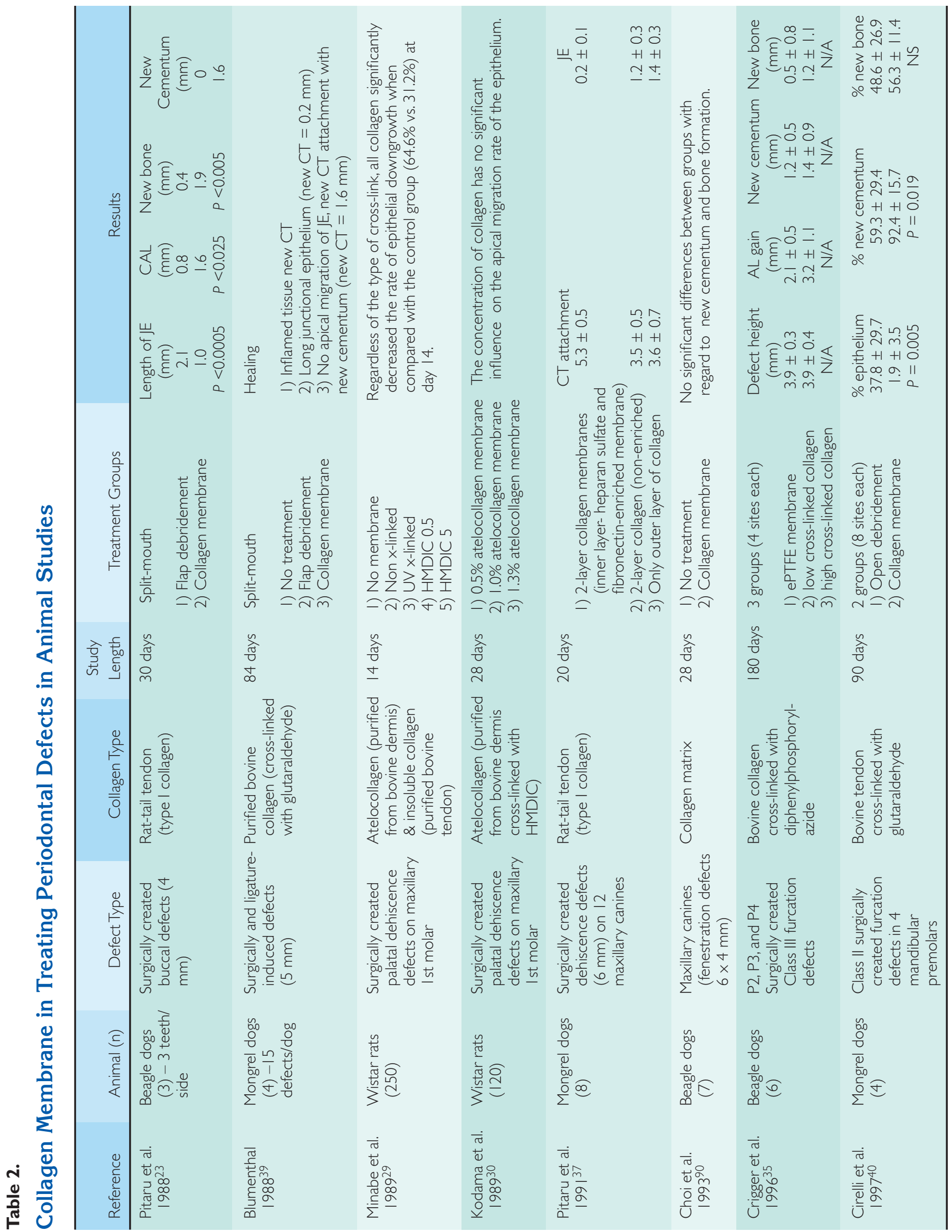


Table 3.

\section{Collagen Membrane in Treating Intrabony Defects in Humans}

\begin{tabular}{|c|c|c|c|c|c|c|c|c|}
\hline Reference & N (mean age) & Collagen Type & Study Length & Treatment Groups & $\begin{array}{l}\text { Mean Initial } \\
\text { PD (mm) }\end{array}$ & $\begin{array}{c}\text { Mean PD } \\
\text { Reduction }(\mathrm{mm})\end{array}$ & $\begin{array}{l}\text { Mean CAL } \\
\text { Gain }(\mathrm{mm})\end{array}$ & $\begin{array}{c}\text { Defect Fill } \\
(\%)\end{array}$ \\
\hline $\begin{array}{l}\text { Chung et al. } \\
1990^{42}\end{array}$ & $\begin{array}{l}15 \text { patients } \\
(21-39 \text { years }) \\
\text { with } 20 \\
\text { paired defects }\end{array}$ & $\begin{array}{l}\text { Cross-linked bovine } \\
\text { type I collagen }\end{array}$ & $\begin{array}{l}12 \text { months } \\
\text { (reentry) }\end{array}$ & $\begin{array}{l}\text { Split-mouth design } \\
\text { I) Flap } \\
\text { debridement } \\
\text { 2) Collagen } \\
\text { membrane }\end{array}$ & $\begin{array}{c}5.4 \pm 1.9 \\
6.0 \pm 2.2 \\
\text { NS }\end{array}$ & $\begin{array}{l}\text { NA } \\
\text { N/A }\end{array}$ & $\begin{array}{c}-0.7 \pm 0.9 \\
0.6 \pm 0.6 \\
P<0.01\end{array}$ & $\begin{array}{l}\text { N/A } \\
\text { N/A }\end{array}$ \\
\hline $\begin{array}{l}\text { Quteish and } \\
\text { Dolby } \\
1992^{31}\end{array}$ & $\begin{array}{l}19 \text { patients } \\
\text { (N/A) with } \\
52 \text { matched } \\
\text { defects }\end{array}$ & $\begin{array}{l}\text { Irradiated } \\
\text { glutaraldehyde } \\
\text { human type I } \\
\text { collagen extracted } \\
\text { from placenta }\end{array}$ & 6 months & $\begin{array}{l}\text { Split-mouth design } \\
\text { I) Flap } \\
\text { debridement } \\
\text { 2) Collagen } \\
\text { membrane }\end{array}$ & $\begin{array}{c}5.8 \pm 1.4 \\
6.4 \pm 1.9 \\
\text { NS }\end{array}$ & $\begin{array}{c}2.2 \\
4.2 \\
P<0.000 \text { I }\end{array}$ & $\begin{array}{c}2.3 \\
4.0 \\
P<0.000 \mid\end{array}$ & $\begin{array}{l}\text { N/A } \\
\text { N/A }\end{array}$ \\
\hline $\begin{array}{l}\text { al-Arrayed } \\
\text { et al. } \\
1995^{43}\end{array}$ & $\begin{array}{l}\text { I } 4 \text { patients } \\
\text { (43.7 years) }\end{array}$ & $\begin{array}{l}\text { Cross-linked human } \\
\text { type I collagen }\end{array}$ & 6 months & $\begin{array}{l}\text { Split-mouth design } \\
\text { 1) Open flap } \\
\text { debridement } \\
\text { 2) Collagen }\end{array}$ & $\begin{array}{l}6.4 \\
6.7 \\
\text { NS }\end{array}$ & $\begin{array}{c}2.8 \\
\\
4.3 \\
P<0.05\end{array}$ & $\begin{array}{c}3.7 \\
3.9 \\
P<0.05\end{array}$ & $\begin{array}{l}\text { N/A } \\
\text { N/A }\end{array}$ \\
\hline $\begin{array}{l}\text { Mattson et } \\
\text { al. } 1995^{44}\end{array}$ & $\begin{array}{l}9 \text { patients } \\
\text { (49.7 years) }\end{array}$ & $\begin{array}{l}\text { Type I bovine } \\
\text { collagen }\end{array}$ & $\begin{array}{l}12 \text { months } \\
\text { (reentry) }\end{array}$ & $\begin{array}{l}\text { Split-mouth design, } \\
\text { but not balanced } \\
\text { for severity } \\
\text { 1) Open flap } \\
\text { debridement } \\
\text { 2) Collagen }\end{array}$ & $\begin{array}{l}6.6 \pm 1.2 \\
7.2 \pm 0.9\end{array}$ & $\begin{array}{l}2.2 \pm 1.8 \\
3.1 \pm 1.6\end{array}$ & $\begin{array}{l}0.4 \pm 2.0 \\
2.4 \pm 2.1\end{array}$ & $\begin{array}{l}28 \\
77\end{array}$ \\
\hline $\begin{array}{l}\text { Benquét et } \\
\text { al. } 199745\end{array}$ & $\begin{array}{l}52 \text { AP patients } \\
\text { and } 16 \text { RPP } \\
\text { patients }\end{array}$ & $\begin{array}{l}\text { DPPA cross-linked } \\
\text { bovine type I } \\
\text { collagen }\end{array}$ & 6 months & $\begin{array}{l}\text { One defect/patient } \\
\text { I) Adult } \\
\text { periodontitis } \\
\text { 2) RPP }\end{array}$ & $\begin{array}{l}9.6 \pm 2.0 \\
8.3 \pm 1.6\end{array}$ & $\begin{array}{l}5.5 \pm 2.4 \\
4.1 \pm 2.6\end{array}$ & $\begin{array}{l}3.6 \pm 2.2 \\
2.6 \pm 3.0\end{array}$ & $\begin{array}{l}\text { N/A } \\
\text { N/A }\end{array}$ \\
\hline $\begin{array}{l}\text { Mattson } \\
\text { et al. } \\
199946\end{array}$ & $\begin{array}{l}19 \text { patients } \\
\text { (50.4 years) } \\
\text { with at least } \\
\text { one 2- to 3- } \\
\text { walled } \\
\text { interproximal } \\
\text { defect }\end{array}$ & $\begin{array}{l}\text { Cross-linked } \\
\text { bovine tendon } \\
\text { (type I collagen) }\end{array}$ & $\begin{array}{l}6 \text { months } \\
\text { (reentry) }\end{array}$ & $\begin{array}{l}\text { 1) Collagen } \\
\text { membrane } \\
(n=\mid I) \\
\text { 2) PGA/PLA } \\
\text { copolymer } \\
\text { membrane } \\
(n=\mid 2)\end{array}$ & $\begin{array}{c}6.14 \pm 2.02 \\
\text { NS }\end{array}$ & $\begin{array}{c}3.55 \pm 2.47 \\
\text { NS }\end{array}$ & $\begin{array}{l}2.58 \pm 1.9 \\
2.77 \pm 2.13 \\
\text { NS }\end{array}$ & $\begin{array}{c}1.9 \pm 1.9 \\
\text { NS }\end{array}$ \\
\hline
\end{tabular}

RPP—rapidly progressive periodontitis.

of those of the actual intrabony defects. With this analytical approach, little or no improvement from shallow PD sites may mask the actual improvement observed on the treated defect sites. Therefore, it is not surprising that the results from this study showed less attachment level gain than other studies. Nevertheless, the majority of these studies showed significantly greater clinical improvements when collagen membranes were used.

Parodi et al. ${ }^{48}$ reported a human histological biopsy of a mesial circumferential defect on a maxillary molar treated with DPPA cross-linked collagen membrane containing $4 \%$ chondroitin-4-sulfate. At 5 months, a clinical attachment gain of $4 \mathrm{~mm}$ was observed. Histological observation demonstrated highly vascularized periodontal ligament, and new cementum with an active cementoblast front and new bone formation up to the crestal level. Moreover, no sign of inflammation was noted. It was concluded that collagen membranes can enhance partial periodontal regeneration in humans. Evans et al. ${ }^{49}$ used meta-analysis to analyze GTR articles published between 1994 to early 1996. The mean PD reduction and CAL gain with a GTR procedure utilizing collagen membranes were 4.1 $\mathrm{mm}$ and $4.0 \mathrm{~mm}$, respectively. These results were comparable to those of studies using ePTFE mem- 
Table 4.

\section{Combined Use of Collagen Membrane and Other Materials in Humans}

\begin{tabular}{|c|c|c|c|c|c|c|c|c|}
\hline Reference & N (mean age) & Collagen Type & Study Length & Treatment Groups & $\begin{array}{l}\text { Mean Initial } \\
\text { PD (mm) }\end{array}$ & $\begin{array}{c}\text { Mean PD } \\
\text { Reduction (mm) }\end{array}$ & $\begin{array}{l}\text { Mean CAL } \\
\text { Gain }(\mathrm{mm})\end{array}$ & $\begin{array}{c}\text { Defect Fill } \\
(\%)\end{array}$ \\
\hline $\begin{array}{l}\text { Blumenthal } \\
\text { and } \\
\text { Steinberg } \\
1990^{50}\end{array}$ & $\begin{array}{l}10 \text { patients } \\
\text { (34-57 years) } \\
\text { with } 71 \text { defects }\end{array}$ & $\begin{array}{l}\text { Purified bovine } \\
\text { collagen, allogenic } \\
\text { freeze-dried bone } \\
\text { \& microfibrillar } \\
\text { collagen gel }\end{array}$ & $\begin{array}{l}12 \text { months } \\
\text { (reentry) }\end{array}$ & $\begin{array}{l}\text { 1) Open } \\
\text { debridement } \\
\text { 2) Membrane alone } \\
\text { 3) Bone graft alone } \\
\text { 4) Bone and } \\
\text { collagen gel } \\
\text { 5) Bone, collagen } \\
\text { gel, and membrane }\end{array}$ & $\begin{array}{l}\text { N/A } \\
\text { N/A } \\
\text { N/A } \\
\text { N/A } \\
\text { N/A }\end{array}$ & $\begin{array}{l}1.5 \pm 0.2 \\
2.0 \pm 0.1 \\
2.0 \pm 0.1 \\
2.6 \pm 0.1 \\
2.7 \pm 0.1\end{array}$ & $\begin{array}{l}0.8 \pm 0.2 \\
1.2 \pm 0.1 \\
1.4 \pm 0.1 \\
1.9 \pm 0.2 \\
2.0 \pm 0.1\end{array}$ & $\begin{array}{l}5.9 \pm 1.5 \\
30.6 \pm 2.8 \\
47.7 \pm 2.7 \\
48.5 \pm 3.5 \\
62.7 \pm 2.5\end{array}$ \\
\hline $\begin{array}{l}\text { Dowell } \\
\text { et al. } \\
\text { 1995 }\end{array}$ & $\begin{array}{l}16 \text { patients } \\
\text { (43.7 } \pm 4 \\
\text { years) with } \\
78 \text { bilateral } \\
\text { defects }\end{array}$ & $\begin{array}{l}\text { Cross-linked human } \\
\text { type I collagen } \\
\text { with or without } \\
\text { metronidazole } \\
\left(8 \mathrm{mg} / \mathrm{cm}^{2}\right)\end{array}$ & 6 months & $\begin{array}{l}\text { Split-mouth design } \\
\text { I) Collagen } \\
\text { membrane } \\
\text { 2) Metronidazole- } \\
\text { impregnated } \\
\text { collagen }\end{array}$ & $\begin{array}{l}6.8 \\
6.7 \\
\text { NS }\end{array}$ & $\begin{array}{c}4.3 \pm 0.9 \\
4.3 \pm 0.9 \\
\text { NS }\end{array}$ & NS & $\mathrm{N} / \mathrm{A}$ \\
\hline $\begin{array}{l}\text { Chen } \\
\text { et al. } \\
1995^{52}\end{array}$ & $\begin{array}{l}10 \text { patients } \\
\text { (26-62 years) } \\
\text { with bilateral } \\
\text { defects }\end{array}$ & $\begin{array}{l}\text { Cross-linked } \\
\text { bovine tendon } \\
\text { with or without } \\
\text { DFDBA }\end{array}$ & $\begin{array}{l}6 \text { to } 12 \text { months } \\
\text { (reentry) }\end{array}$ & $\begin{array}{l}\text { Split-mouth design } \\
\text { I) Collagen } \\
\text { membrane } \\
\text { 2) Collagen } \\
\text { membrane with } \\
\text { DFDBA }\end{array}$ & $\begin{array}{c}7.4 \pm 0.4 \\
7.6 \pm 0.4 \\
\text { NS }\end{array}$ & $\begin{array}{c}3.2 \pm 0.4 \\
3.4 \pm 0.4 \\
\text { NS }\end{array}$ & $\begin{array}{c}2.0 \pm 0.4 \\
2.3 \pm 0.5 \\
\text { NS }\end{array}$ & $\begin{array}{l}39.6 \\
35.4 \\
\text { NS }\end{array}$ \\
\hline $\begin{array}{l}\text { Benqué } \\
\text { et al. } \\
1997^{53}\end{array}$ & $\begin{array}{l}43 \text { AP patients } \\
(49.2 \pm 9.9 \\
\text { years) and I } 4 \\
\text { RPP patients } \\
\text { (38 } \pm 4.7 \\
\text { years) with } \\
\text { deep 2-wall } \\
\text { bony defects }\end{array}$ & $\begin{array}{l}\text { DPPA cross-linked } \\
\text { bovine type I } \\
\text { collagen with or } \\
\text { without } \\
\text { hydroxyapatite/ } \\
\text { collagen/ } \\
\text { chondroitin } \\
\text { sulfate grafting } \\
\text { material }\end{array}$ & 6 months & $\begin{array}{l}\text { One defect/patient } \\
\text { I) AP patients } \\
\text { 2) RPP patients }\end{array}$ & $\begin{array}{l}9.5 \pm 2.0 \\
8.2 \pm 1.8\end{array}$ & $\begin{array}{l}6.1 \pm 2.1 \\
4.7 \pm 1.2 \\
P<0.05\end{array}$ & $\begin{array}{l}4.2 \pm 2.1 \\
3.0 \pm 1.6 \\
P<0.05\end{array}$ & $\begin{array}{l}\text { N/A } \\
\text { N/A }\end{array}$ \\
\hline
\end{tabular}

AP-adult periodontitis.

RPP_rapidly progressive periodontitis.

branes $(5.1 \mathrm{~mm}$ and $3.6 \mathrm{~mm}$, respectively). The authors also reported that there were no statistically significant differences in clinical outcomes among various GTR membranes (ePTFE, collagen, collagen plus bone graft, polylactic acid/polyglycolic acid copolymer [PLA/PGA], and rubber dam). However, it should be remembered that even with the best results ever reported, none of these studies demonstrated a complete fill of periodontal defects.

\section{Combined Use of Collagen Membrane and Other Materials}

Several attempts have been made to enhance regeneration outcomes by combining/adding other materials. These include a combination of collagen membranes and bone grafting materials, metronidazoleimpregnated collagen, fibronectin and heparan sulfate- enriched collagen, and a combination of collagen membrane and collagen gel (Table 4). ${ }^{37,50-53}$ These techniques mainly aim to provide space maintenance and to recruit cells with regenerative potential. It is believed that bone grafting materials not only maintain the space, but also provide an osteoinductive and/or osteoconductive capacity. In 1990, Blumenthal and Steinberg ${ }^{50}$ reported statistically significant improvement in defect fill with the use of freeze-dried bone and microfibrillar collagen gel $^{\ddagger}$ in addition to collagen barrier. However, it should be noted that this study was conducted in only 10 patients (71 defects) with 5 different treatment groups. Also, the original PD or description of defect morphology is lacking. Chen et

† Zyderm, Collagen Corp., Palo Alto, CA. 
al., ${ }^{52}$ in a randomized split-mouth design clinical trial, showed that demineralized freeze-dried bone allograft (DFDBA) had no additional benefit to the GTR therapy with collagen membrane alone. The use of grafting material containing hydroxyapatite, collagen, and chondroitin-sulfate $\S$ in addition to the collagen membrane was also studied by Benqué et al. ${ }^{53}$ The authors attempted to treat 2-wall intrabony defects in both chronic adult periodontitis and rapidly progressive periodontitis patients. The results indicated that this combined technique significantly improved PD and CAL in both patient groups. In addition, there were statistically significant greater PD reduction and CAL gain in chronic adult periodontitis patients than rapidly progressive periodontitis patients. However, this study lacked a negative control (open debridement), positive control (membrane alone), and placebo control (membrane plus gel carrier) groups; therefore, it is inconclusive whether the spacer or its components accounted for the improvement.

In addition to space maintaining materials, antibiotic-impregnated collagen membranes have also been utilized to reduce the risk of bacterial contamination. Dowell et al., ${ }^{51}$ in a double-blinded, randomized, splitmouth design clinical trial, failed to demonstrate the extra benefit of metronidazole-impregnated collagen over the collagen membrane alone.

Class II furcation defects. It is generally agreed that treatment of furcation defects is more complicated and challenging than that of non-furcated

$\S$ Biostite, Coletica Corp., Lyon, France.

Table 5.

\section{Collagen Membrane in Treating Class II Furcation Defects in Humans}

\begin{tabular}{|c|c|c|c|c|c|c|c|c|c|}
\hline \multirow[b]{2}{*}{ Reference } & \multirow[b]{2}{*}{$N($ mean age $)$} & \multirow{2}{*}{$\begin{array}{l}\text { Collagen Type } \\
\text { \& Additional } \\
\text { Materials }\end{array}$} & \multirow[b]{2}{*}{$\begin{array}{l}\text { Study } \\
\text { Length }\end{array}$} & \multirow[b]{2}{*}{ Treatment Groups } & \multicolumn{4}{|c|}{ Results } & \multirow{2}{*}{$\begin{array}{l}\% \mathrm{Cl} \| \\
\rightarrow \mathrm{Cl} I \\
\text { Defects }\end{array}$} \\
\hline & & & & & $\begin{array}{c}\text { Initial } \\
\text { V-Depth }\end{array}$ & $\begin{array}{c}\text { Initial } \\
\text { H-Depth }\end{array}$ & $\begin{array}{l}\text { V-Defect } \\
\text { Fill }\end{array}$ & $\begin{array}{l}\text { H-Defect } \\
\text { Fill }\end{array}$ & \\
\hline $\begin{array}{l}\text { Paul et al. } \\
1992^{22}\end{array}$ & $\begin{array}{l}7 \text { patients ( } 51.7 \\
\text { years) with bilateral } \\
\text { defects }\end{array}$ & Type I bovine collagen & $\begin{array}{l}6 \text { months } \\
\text { (reentry) }\end{array}$ & $\begin{array}{l}\text { Split-mouth design } \\
\text { 1) Flap debridement } \\
\text { 2) Collagen membrane }\end{array}$ & $\begin{array}{c}6.1 \pm 1.8 \\
6.4 \pm 1.9 \\
\text { NS }\end{array}$ & $\begin{array}{c}3.9 \pm 2.1 \\
4.7 \pm 2.0 \\
\text { NS }\end{array}$ & $\begin{array}{c}0.4 \pm 0.8 \\
0.7 \pm 0.9 \\
\text { NS }\end{array}$ & $\begin{array}{l}0.0 \pm 0.9 \\
0.9 \pm 0.9 \\
P<0.05\end{array}$ & N/A \\
\hline $\begin{array}{l}\text { Van Swol } \\
\text { et al. } \\
1993^{60}\end{array}$ & $\begin{array}{l}38 \text { patients }(3|-7| \\
\text { years) }\end{array}$ & $\begin{array}{l}\text { Chemical cross-linked } \\
\text { purified bovine } \\
\text { dermal collagen }\end{array}$ & $\begin{array}{l}3 \text { months } \\
\text { (reentry) }\end{array}$ & $\begin{array}{l}\text { One defect/patient } \\
\text { I) Flap debridement } \\
\text { (10) } \\
\text { 2) Collagen membrane } \\
\text { (28) }\end{array}$ & $\begin{array}{c}7.1 \pm 1.6 \\
6.9 \pm 1.6 \\
\text { NS }\end{array}$ & $\begin{array}{c}5.7 \pm 2.5 \\
5.1 \pm 1.4 \\
\text { NS }\end{array}$ & $\begin{array}{c}0.7 \\
1.7 \\
P<0.05\end{array}$ & $\begin{array}{c}0.7 \\
2.3 \\
P<0.000 \text { । }\end{array}$ & $\begin{array}{c}>50 \% \text { H-Fill } \\
0 / 10 \\
5 / 28\end{array}$ \\
\hline $\begin{array}{l}\text { Blumenthal } \\
1993^{62}\end{array}$ & $\begin{array}{l}12 \text { patients ( } 3 \mid-80 \\
\text { years) with paired } \\
\text { buccal Class II } \\
\text { mandibular molar } \\
\text { furcation defects }\end{array}$ & $\begin{array}{l}\text { Chemical cross-linked } \\
\text { purified bovine } \\
\text { dermal collagen }\end{array}$ & $\begin{array}{l}12 \text { months } \\
\text { (reentry) }\end{array}$ & $\begin{array}{l}\text { Split-mouth design } \\
\text { 1) ePTFE membrane } \\
\text { 2) Collagen membrane }\end{array}$ & $\begin{array}{l}6.2 \pm 2.2 \\
7.00 \pm 2.2 \\
\text { NS }\end{array}$ & $\begin{array}{c}4.9 \pm 0.7 \\
5.3 \pm 1.3 \\
\text { NS }\end{array}$ & $\begin{array}{c}1.0 \pm 1.0 \\
1.6 \pm 1.2 \\
\text { NS }\end{array}$ & $\begin{array}{l}1.7 \pm 0.5 \\
2.5 \pm 0.7 \\
P<0.05\end{array}$ & $N / A$ \\
\hline $\begin{array}{l}\text { Black et al. } \\
1994^{63}\end{array}$ & $\begin{array}{l}\text { I } 3 \text { patients ( } 43.2 \\
\text { years) with a pair } \\
\text { of comparable Class } \\
\text { II molar defects }\end{array}$ & $\begin{array}{l}\text { Cross-linked bovine } \\
\text { tendon type I collagen } \\
\text { s }\end{array}$ & 6 months & $\begin{array}{l}\text { Split-mouth design } \\
\text { 1) ePTFE membrane } \\
\text { 2) Collagen membrane }\end{array}$ & $\begin{array}{l}5.3 \pm 1.6 \\
5.00 \pm 1.8 \\
\text { NS }\end{array}$ & $\begin{array}{c}4.3 \pm 2.0 \\
4.4 \pm 1.5 \\
\text { NS }\end{array}$ & $\begin{array}{c}1.1 \pm 0.8 \\
1.4 \pm 1.7 \\
\mathrm{NS}\end{array}$ & $\begin{array}{l}0.8 \pm 2.2 \\
1.5 \pm 2.0\end{array}$ & N/A \\
\hline $\begin{array}{l}\text { Wang } \\
\text { et al. } \\
1994^{20}\end{array}$ & $\begin{array}{l}12 \text { patients ( } 46.4 \\
\text { years) with bilateral } \\
\text { mandibular } \\
\text { furcation defects }\end{array}$ & $\begin{array}{l}\text { Cross-linked type I } \\
\text { | bovine collagen }\end{array}$ & $\begin{array}{l}12 \text { months } \\
\text { (reentry) }\end{array}$ & $\begin{array}{l}\text { Split-mouth design } \\
\text { 1) Flap debridement } \\
\text { 2) Collagen membrane }\end{array}$ & $\begin{array}{l}4.2 \pm 2.2 \\
4.2 \pm 1.7 \\
\text { NS }\end{array}$ & $\begin{array}{c}5.6 \pm 2.7 \\
6.0 \pm 2.7 \\
\text { NS }\end{array}$ & $\begin{array}{l}1.5 \pm 0.3 \\
2.8 \pm 0.4 \\
P<0.05\end{array}$ & $\begin{array}{c}1.1 \pm 0.6 \\
2.0 \pm 0.4 \\
\text { NS }\end{array}$ & $\begin{array}{c}>50 \% \mathrm{H} \text {-Fill } \\
4 / 12 \\
3 / 12\end{array}$ \\
\hline $\begin{array}{l}\text { Yukna and } \\
\text { Yukna } \\
1996^{64}\end{array}$ & $\begin{array}{l}59 \text { patients ( } 46.8 \\
\text { years) from } 7 \\
\text { centers }\end{array}$ & $\begin{array}{l}\text { Type I bovine tendon } \\
\text { collagen }\end{array}$ & $\begin{array}{c}6 \text { to } 12 \\
\text { months } \\
\text { (mean } \\
\mid 1.1 \text { ) } \\
\text { (reentry) }\end{array}$ & $\begin{array}{l}\text { Split-mouth design } \\
\text { I) Flap debridement } \\
\text { (27) } \\
\text { Collagen membrane } \\
\text { (27) } \\
\text { 2) ePTFE membrane } \\
\text { (32) } \\
\text { Collagen membrane } \\
\text { (32) }\end{array}$ & $\begin{array}{l}3.2 \pm 1.6 \\
3.6 \pm 1.7 \\
1.9 \pm 2.5 \\
1.3 \pm 1.0\end{array}$ & $\begin{array}{l}5.3 \pm 2.0 \\
5.0 \pm 2.0 \\
4.3 \pm 1.7 \\
4.7 \pm 1.6\end{array}$ & $\begin{array}{c}0.8 \pm 1.5 \\
1.7 \pm 1.6 \\
\text { N/A } \\
\text { N/A }\end{array}$ & $\begin{array}{l}1.1 \pm 2.0 \\
2.0 \pm 1.7 \\
1.7 \pm 2.2 \\
1.7 \pm 1.9\end{array}$ & $\begin{array}{c}\text { \% improved } \\
\text { defects } \\
7 \\
44 \\
53\end{array}$ \\
\hline
\end{tabular}


defects because of the complexities of tooth anatomy including root concavities, ${ }^{54}$ cervical enamel projections, ${ }^{55}$ bifurcation ridges, ${ }^{56}$ accessory canals, ${ }^{57}$ and small entrance access. ${ }^{58}$ Nonetheless, attempts have been made to regenerate lost periodontium in furcation defects through varieties of approaches. Table 5 lists studies that have utilized collagen membranes to promote regeneration in furcated molars. In 1988, Garrett et al. ${ }^{59}$ used a collagenous membrane, freezedried dura mater, for treating furcation-involved teeth. The results, however, showed less favorable outcomes than coronally positioned flaps with allograft-treated sites. Paul et al. ${ }^{22}$ showed minor improvement in horizontal defect fill, with no difference in vertical bone fill when comparing a collagen membrane-treated group to an open debridement control group. In contrast, Van Swol et al. ${ }^{60}$ and Wang et al. ${ }^{20}$ reported significantly greater defect fills in a collagen-treated group than an open debridement control group. This may be explained by the differences in the protocol; i.e., collagen membranes were intentionally left supragingivally in the former study while primary coverage was attempted in the latter studies. Since primary coverage is a key factor in periodontal regen- eration and premature membrane exposure often leads to bacterial colonization and compromised clinical outcomes, ${ }^{26,28,61}$ it is possible that the unfavorable outcomes from Garrett et al. ${ }^{59}$ and Paul et al. ${ }^{22}$ were partly the consequences of exposed membranes. When comparing the use of collagen material to nonabsorbable ePTFE membrane, comparable clinical results were noted in treating Class II furcation defects. ${ }^{62-64}$ Nevertheless, it should be noted that only $14 \%$ of these treated sites showed complete furcation fill. ${ }^{64}$ Hence, further studies or attempts are still needed to identify the best methods/materials for furcation defect regeneration/repair.

\section{Root Coverage Procedures}

Different techniques have been used to replace lost, damaged, or diseased gingival tissues. Traditionally, multiple procedures are often needed to achieve optimal esthetic results. These include lateral pedicle graft, free gingival graft, and subepithelial connective tissue graft. However, these procedures often require a second surgical site for harvesting grafts, and may cause postsurgical bleeding and patient discomfort. Recently, attempts have been made to achieve root coverage

\section{Table 6.}

\section{Collagen Membrane in Root Coverage Procedures}

\begin{tabular}{|c|c|c|c|c|c|c|c|c|c|}
\hline \multirow[b]{2}{*}{ Reference } & \multirow[b]{2}{*}{$\begin{array}{l}N \text { (mean } \\
\text { age) }\end{array}$} & \multirow[b]{2}{*}{ Collage Type } & \multirow[b]{2}{*}{$\begin{array}{l}\text { Study } \\
\text { Length }\end{array}$} & \multirow[b]{2}{*}{ Defect Type } & \multicolumn{5}{|c|}{ Results } \\
\hline & & & & & $\begin{array}{l}\text { Mean Initial } \\
\text { Recession } \\
\text { Depth (mm) }\end{array}$ & $\begin{array}{l}\text { Gain in } \\
\text { CAL } \\
(\mathrm{mm})\end{array}$ & $\begin{array}{l}\text { Mean Root } \\
\text { Coverage } \\
(\mathrm{mm})\end{array}$ & $\begin{array}{l}\text { Mean \% } \\
\text { Root } \\
\text { Coverage }\end{array}$ & $\begin{array}{c}\text { \% Sites With } \\
\text { Complete } \\
\text { Coverage }\end{array}$ \\
\hline $\begin{array}{l}\text { Shieh et al. } \\
1997^{74}\end{array}$ & $\begin{array}{l}10 \text { patients } \\
(40.7 \text { years })\end{array}$ & $\begin{array}{l}\text { Cross-linked type I } \\
\text { bovine collagen }\end{array}$ & $\begin{array}{c}6 \\
\text { months }\end{array}$ & $\begin{array}{l}\text { Miller Class I or II buccal } \\
\text { marginal tissue } \\
\text { recession } \geq 2.5 \mathrm{~mm}\end{array}$ & $3.2 \pm 0.3$ & $\begin{array}{l}1.3 \pm 0.5 \\
P<0.05\end{array}$ & $\begin{array}{l}1.7 \pm 0.2 \\
P<0.05\end{array}$ & $\begin{array}{l}51.6 \pm 6.8 \\
P<0.05\end{array}$ & 10 \\
\hline $\begin{array}{l}\text { Zahedi } \\
\text { et al. } \\
1998^{75}\end{array}$ & $\begin{array}{l}\text { I patients } \\
\text { (46.7 years) }\end{array}$ & $\begin{array}{l}\text { DPPA cross-linked } \\
\text { bovine } 96 \% \text { type I } \\
\text { collagen with } 4 \% \\
\text { chondroitin-4-sulfate }\end{array}$ & $\begin{array}{c}24 \\
\text { months }\end{array}$ & Miller Class I & $3.7 \pm 1.4$ & $\begin{array}{l}3.5 \pm 1.3 \\
P<0.0001\end{array}$ & $2.9 \pm 1.1$ & $\begin{array}{c}82.2 \pm 23.2 \\
P<0.0001\end{array}$ & 53 \\
\hline $\begin{array}{l}\text { Özcan } \\
\text { et al. } \\
1997^{76}\end{array}$ & $\begin{array}{l}\text { I0 patients } \\
\text { (38.5 years) } \\
\text { with } 28 \\
\text { Miller's Class I } \\
\text { or II ( } \geq 3 \mathrm{~mm} \\
\text { recession } \\
\text { depth) } \\
\text { defects }\end{array}$ & $\begin{array}{l}\text { Tutoplast fascia } \\
\text { temporalis }\end{array}$ & $\begin{array}{c}6 \\
\text { months }\end{array}$ & $\begin{array}{l}\text { 1) Coronally positioned f } \\
\text { 2) Collagen membrane, } \\
\text { fibrin/fibronectin and } \\
\text { tetracycline root } \\
\text { conditioning }\end{array}$ & $\begin{array}{r}\text { flap } 4.1 \\
4.3\end{array}$ & $\begin{array}{c}2.9 \\
4.2 \\
P<0.001\end{array}$ & $\begin{array}{l}2.8 \\
3.1 \\
\text { NS }\end{array}$ & $\begin{array}{l}68.6 \\
71.7 \\
\text { NS }\end{array}$ & $\begin{array}{l}\text { N/A } \\
\text { N/A }\end{array}$ \\
\hline $\begin{array}{l}\text { Wang } \\
\text { et al. } \\
199977\end{array}$ & $\begin{array}{l}\text { I6 patients } \\
\text { (40.6 years) } \\
\text { with paired } \\
\text { Miller's Class I } \\
\text { or II ( } \geq 3 \mathrm{~mm} \\
\text { recession } \\
\text { depth) defects }\end{array}$ & $\begin{array}{l}\text { Newly designed } \\
\text { double-thickness } \\
\text { membrane } \\
\text { (bovine tendon) } \\
\text { ts }\end{array}$ & $\begin{array}{c}6 \\
\text { months }\end{array}$ & $\begin{array}{l}\text { Split-mouth design } \\
\text { I) Subepithelial } \\
\text { connective tissue graft } \\
\text { 2) Newly designed } \\
\text { collagen membrane }\end{array}$ & $\begin{array}{c}3.4 \pm 1.0 \\
3.7 \pm 1.1 \\
\mathrm{NS}\end{array}$ & $\begin{array}{l}2.3 \pm 1.0 \\
2.8 \pm 0.7 \\
\text { NS }\end{array}$ & $\begin{array}{c}2.8 \pm 0.8 \\
2.5 \pm 0.7 \\
\text { NS }\end{array}$ & $\begin{array}{c}84 \pm 25 \\
73 \pm 26 \\
\text { NS }\end{array}$ & $\begin{array}{l}43.7 \\
43.7 \\
\text { NS }\end{array}$ \\
\hline
\end{tabular}


using surgical techniques based on the principles of GTR. ${ }^{65-68}$ Similar results were reported when GTRbased techniques were compared to traditional root coverage procedures. ${ }^{69-72}$ However, GTR therapy offers an additional benefit through its potential of achieving new attachment formation along the previously denuded root surface, while it rarely occurred when a subepithelial connective tissue graft was utilized. ${ }^{73}$

Collagen membranes have recently been used in root coverage procedures (Table 6). While Shieh et al. ${ }^{74}$ gained $51.6 \%$ root coverage after 6 months, Zahedi et al. ${ }^{75}$ reported mean root coverage of $82.2 \%$ after 2 years. Since neither of these studies included a control group, it is inconclusive if the GTR-based procedure is comparable to the conventional procedures. In 1997, Özcan et al. ${ }^{76}$ compared the use of collagen membranes containing fibrin/fibronectin plus tetracycline root conditioning to coronally positioned flaps. They reported no significant differences between groups with regard to mean root coverage. However, significantly more gain in clinical attachment was found in the collagen membrane group compared to the coronally positioned flap (4.21 mm versus $2.86 \mathrm{~mm}$ ). Wang et al. ${ }^{77}$ compared a conventional subepithelial connective tissue graft to a GTR-based procedure using a newly designed double-thickness collagen membrane (prepared from cross-linked bovine tendon and supplied in individual $20 \times 15 \times 0.4-0.5 \mathrm{~mm}$ pieces) in 16 patients with bilateral Miller's Class I or II (gingival recession $\geq 3.0 \mathrm{~mm}$ ) recession defects. Data from this study indicated that the GTR-based procedure resulted in statistically significant $(P<0.05)$ improvements in recession depth, CAL, and width of keratinized and attached gingiva compared to baseline. Moreover, the results were comparable to those achieved from subepithelial connective tissue grafts, with mean root coverage of $73 \%$ and $84 \%$ for the newly designed collagen membrane and conventional connective tissue graft, respectively. Recently, Kimble et al. ${ }^{78}$ studied the effect of adjunctive bone graft placement during GTR-based root coverage procedures. Twenty patients with a Miller Class I or II buccal recession defect $\geq 2.5 \mathrm{~mm}$ were randomly treated with either type I collagen membrane alone or type I collagen membrane plus DFDBA. Preliminary results from this study indicated both forms of treatment resulted in statistically significant $(P<0.05)$ reduction of recession depths $(69 \%$ for membrane alone and $79 \%$ for membrane plus DFDBA). It was concluded that there is a tendency for an additional benefit of bone graft for percent root coverage; however, no statistically significant difference was found between groups.

In summary, data from available resources indicate that GTR-based procedures and subepithelial connective tissue grafts are clinically equivalent. In addition, using a barrier may also enhance more clinical attachment gain. ${ }^{76}$ It should also be noted that with the GTR-based procedure, adequate flap thickness $(\geq 0.5 \mathrm{~mm}$ in the defect area) seems to have a great influence in improving the percent root coverage as reported by Harris ${ }^{70}$ (26.7\% versus $95.9 \%$ root coverage in thin and thick tissue, respectively). Therefore, careful case selection is crucial for the success of this procedure.

\section{Guided Bone Regeneration (GBR)}

Implants have slowly emerged as an alternative procedure for replacing missing teeth. Ridge deficiency or inadequate bone height/width often compromises the overall treatment plan. Bone augmentation using GTR principles has become a common means to resolve these concerns. Studies have reported success in using barriers to augment bone prior to or after implant placement. ${ }^{79}$ When selecting an ideal material for GBR, the following requirements must be considered: wound stabilization, space creation and maintenance, protection of the underlying blood clot, and the ability to exclude unwanted tissues or cells (connective tissue and epithelium). Since collagen membranes fulfill all these criteria, it has become one of the materials used in GBR procedures. However, the fast resorption rate of collagen material remains a concern to most clinicians. So far, no study has defined the actual time needed for GBR barriers. Iglhaut et al. ${ }^{80}$ reported that during GTR procedures, bone and/or PDL cell migration reach their peaks in 2 to 7 days after surgery, with a decrease in mitotic activity to almost normal levels by the end of the third week. It could be implied that cells needed for regeneration arrive at the wound sites in around 3 to 4 weeks; therefore, the length of time that the collagen membrane retains its integrity should be sufficient to allow selective cell repopulation.

GBR procedures utilizing collagen membranes have been widely reported (Table 7). ${ }^{81-85}$ In a pilot study, Colangelo et al. ${ }^{81}$ created through and through defects on the lateral aspect of rabbit mandibles and then treated these defects with either a collagen membrane or no-treatment control. The histologic evaluation at 30 days demonstrated a nearly complete continuous layer of lamellar bone with osteoblastic activity in the collagen membrane-treated group compared to only fibrous connective tissue in the control group. Sevor et al. ${ }^{82}$ created buccal dehiscence defects after placement of a total of 48 implants (24 hydroxyapatite [HA]coated and 24 grit-blasted) in 6 dogs. At 8 weeks, the mean defect fill was $80.29 \%$ in the collagen membrane-treated group compared to $38.62 \%$ in the control group.

Contrary to the GTR procedures, additional bone grafting materials for space creation/maintenance tend to improve GBR outcomes. This was supported by 
Table 7.

\section{Collagen Membrane in Guided Bone Regeneration Procedures}

\begin{tabular}{|c|c|c|c|c|c|c|}
\hline Reference & Model (n) & Collagen Type & Study Length & Treatment Groups & \multicolumn{2}{|c|}{$\begin{array}{c}\text { Results } \\
\text { Clinical and Histologic Observations }\end{array}$} \\
\hline $\begin{array}{l}\text { Sevor } \\
\text { et al. } \\
1993^{82}\end{array}$ & $\begin{array}{l}\text { Mongrel dogs } \\
\text { with } \\
\text { surgically } \\
\text { created } \\
\text { buccal } \\
\text { dehiscence } \\
\text { defects } \\
\text { (6 sites/dog) }\end{array}$ & $\begin{array}{l}\text { Highly cross- } \\
\text { linked collagen }\end{array}$ & 1 and 2 months & $\begin{array}{l}\text { Randomly assigned \% } \\
\text { HA-coated implants } \\
\text { (24) } \\
\text { I) No treatment } \\
\text { 2) Collagen } \\
\text { membrane } \\
\text { Grit-blasted implants } \\
\text { (24) } \\
\text { 1) No treatment } \\
\text { 2) Collagen } \\
\text { membrane }\end{array}$ & $\begin{array}{l}\text { \% Defect Fill at } 4 \text { Weeks } \\
\qquad \begin{array}{l}47.11 \pm 23.13 \\
83.13 \pm 3.32\end{array}\end{array}$ & $\begin{array}{l}32.66 \pm 17.61 \\
81.80 \pm 6.11 \\
\\
44.58 \pm 28.73 \\
78.79 \pm 21.70 \\
P<0.007\end{array}$ \\
\hline $\begin{array}{l}\text { Colangelo } \\
\text { et al. } \\
1993^{81}\end{array}$ & $\begin{array}{l}\text { Rabbits with } \\
3 \times 9 \mathrm{~mm} \\
\text { defects on } \\
\text { the lateral } \\
\text { aspect of the } \\
\text { mandible (15) }\end{array}$ & $\begin{array}{l}\text { Cross-linked bovine } \\
\text { tendon type I } \\
\text { collagen }\end{array}$ & I month & $\begin{array}{l}\text { 1) No treatment } \\
\text { control ( } 3 \text { ) } \\
\text { 2) Collagen } \\
\text { membrane (12) }\end{array}$ & \multicolumn{2}{|c|}{$\begin{array}{l}\text { 1) The defects were filled with fibrous } C T \text {. } \\
\text { 2) Layer of lamellar bone was noted with osteoblastic } \\
\text { activity. }\end{array}$} \\
\hline $\begin{array}{l}\text { Zitzmann } \\
\text { et al. } \\
1997^{86}\end{array}$ & $\begin{array}{l}84 \text { exposed } \\
\text { implant } \\
\text { surfaces }(25)\end{array}$ & $\begin{array}{l}\text { Porcine collagen } \\
\text { types I and III }\end{array}$ & 24 months & $\begin{array}{l}\text { Split-mouth design } \\
\text { 1) ePTFE } \\
\text { membrane and } \\
\text { BioOss }(4 I) \\
\text { 2) Collagen } \\
\text { membrane and } \\
\text { BioOss (43) }\end{array}$ & $\begin{array}{c}\text { Mean \% Bone Fill } \\
78 \% \\
\\
92 \% \\
\text { NS }\end{array}$ & \\
\hline $\begin{array}{l}\text { Parodi } \\
\text { et al. } \\
1998^{88}\end{array}$ & $\begin{array}{l}\text { Patients (mean } \\
\text { age } 44.8 \\
\text { years) with } \\
\text { insufficient } \\
\text { ridge (width } \\
<5 \mathrm{~mm} \text { ) } \\
(16)\end{array}$ & $\begin{array}{l}\text { Calfskin collagen } \\
\text { (96\%) with } 4 \% \\
\text { chondroitin-4- } \\
\text { sulfate }\end{array}$ & $\begin{array}{l}7 \text { to } 12 \\
\text { months }\end{array}$ & $\begin{array}{l}\text { Native collagen } \\
\text { sponges were } \\
\text { used to maintain } \\
\text { the space buccally } \\
\text { and lingually. The } \\
\text { membranes were } \\
\text { placed to cover } \\
\text { the sponges. }\end{array}$ & \multicolumn{2}{|c|}{$\begin{array}{l}75 \% \text { of the defects demonstrated sufficient width for } \\
\text { implant placement. Mean increase in the size of the crest } \\
\text { was } 2.5 \mathrm{~mm} \text { ( } 3 \text { to } 5.5 \mathrm{~mm} \text { ). }\end{array}$} \\
\hline $\begin{array}{l}\text { Hürzeler } \\
\text { et al. } \\
1998^{83}\end{array}$ & $\begin{array}{l}\text { Monkeys } \\
\text { (Macaca } \\
\text { fascicularis) } \\
\text { with } \\
\text { surgically } \\
\text { created } \\
\text { defects } \\
(4 \times 4-5 \times \\
20 \mathrm{~mm})(5)\end{array}$ & $\begin{array}{l}\text { Calfskin collagen } \\
\text { (96\%) with } 4 \% \\
\text { chondroitin-4- } \\
\text { sulfate }\end{array}$ & 6 months & $\begin{array}{l}\text { 1) Collagen } \\
\text { membrane } \\
\text { 2) Collagen } \\
\text { membrane and } \\
\text { BioOss } \\
\text { 3) ePTFE } \\
\text { membrane and } \\
\text { BioOss } \\
\text { 4) No membrane contr }\end{array}$ & $P<0.05$ betv & $\begin{array}{l}\text { ne/implant contact } \\
2.2 \pm 0.9 \mathrm{~mm} \\
3.3 \pm 1.9 \mathrm{~mm} \\
3.9 \pm 1.4 \mathrm{~mm} \\
1.4 \pm 0.7 \mathrm{~mm} \\
\text { tween } 1.4 \text { and } 2.3\end{array}$ \\
\hline $\begin{array}{l}\text { Dongieux } \\
\text { et al. } \\
1998^{84}\end{array}$ & $\begin{array}{l}\text { Mongrel dogs } \\
\text { received } 6 \\
\text { monocortical } \\
\text { onlay grafts } \\
\text { on the } \\
\text { mandibles } \\
\text { (4) }\end{array}$ & $\begin{array}{l}\text { Cross-linked } \\
\text { bovine type I } \\
\text { collagen }\end{array}$ & 2.5 months & $\begin{array}{l}\text { Split-mouth design } \\
\text { ( } 2 \text { for each group } \\
\text { in each animal) } \\
\text { 1) Onlay graft alone } \\
\text { 2) Onlay graft with } \\
\text { ePTFE membrane } \\
\text { 3) Onlay graft with } \\
\text { collagen membran }\end{array}$ & $\begin{array}{l}\begin{array}{c}\text { Initial Graft Volume Final Graft Volume } \\
(\mathrm{ml})\end{array} \\
(\mathrm{ml}) \\
0.15 \pm 0.02 \\
0.18 \pm 0.03 \\
0.16 \pm 0.03 \\
\mathrm{NS}\end{array}$ & $\begin{array}{l}\text { Final: Initial Volume } \\
\text { Ratio } \\
\begin{array}{c}0.06 \pm 0.040 .40 \\
0.06 \pm 0.060 .32 \\
0.07 \pm 0.060 .46 \\
\text { NS }\end{array}\end{array}$ \\
\hline
\end{tabular}


Hürzeler et al. ${ }^{83}$ The authors reported collagen membrane combined with bovine HA significantly enhanced bone-to-implant contact when compared to collagen membrane alone, or non-membrane treated control groups. In addition, the results were comparable to those in the ePTFE membrane plus bovine HA group. This was further confirmed by Zitzmann et al. ${ }^{86}$

Collagen membranes have been reported to be superior to non-absorbable membranes with regard to healing. Also, a higher incidence of dehiscence, membrane exposure, and/or premature membrane removal was noted in non-absorbable membranes. ${ }^{81,82,86} \mathrm{Sim}$ ilar to GTR procedures, membrane exposure has a negative impact on the amount of bone regenerated in GBR. Simion et al. ${ }^{87}$ reported significantly less bone gain when the membranes were exposed compared to non-exposed membrane treated sites $(41.6 \%$ versus 96.6\%).

Collagen sponges have also been placed beneath collagen membranes to provide space needed for new bone formation. Parodi et al. ${ }^{88}$ used collagen membranes combined with a collagen sponge to correct 16 cases with insufficient ridges ( $<5 \mathrm{~mm}$ in width). After 7 to 12 months, $75 \%$ of the sites demonstrated sufficient bone volume for implant placement with the mean increase in crestal bone of $2.49 \mathrm{~mm}$. Recently, monocortical onlay grafts were introduced to augment bone prior to implant placement. Dongieux et al. ${ }^{84}$ evaluated the necessity of barrier membranes in addition to onlay grafts in a dog model. This 10 -week splitmouth design demonstrated that neither ePTFE membranes nor collagen membranes have an extra benefit to the onlay graft alone.

To date, the factors that influence bone regeneration are complex and not yet fully understood. Bone regeneration utilizing collagen membranes seems promising. It should also be noted that the available data are from studies within the 1990s. Long-term studies are needed to confirm the success rate of implants placed in regenerated bone.

\section{CONCLUSION}

In conclusion, collagen membranes have been used in both medical and dental (especially periodontal) fields for decades. The benefits of utilizing this absorbable material include promoting wound healing through clot stabilization, wound stability, and hemostasis; enhancing primary wound coverage via its chemotactic ability to attract fibroblasts; and augmenting flap thickness by providing a collagenous scaffold. Cross-linked membranes allow a slower degradation rate, therefore providing more time for selective cells to repopulate the wound areas. Generally, collagen membranes have been proven to significantly enhance periodontal regeneration in various animal studies and human clinical trials; however, none of these studies has shown a complete regeneration. In addition, collagen membranes have been applied to guided bone regeneration and root coverage procedures with comparable success rates to non-absorbable ePTFE membranes and conventional subepithelial connective tissue grafts, respectively. More research is necessary to improve clinical outcomes and achieve true periodontal regeneration. A better understanding of the factors orchestrated in the regeneration process is required to achieve $100 \%$ predictable outcomes in treating osseous defects around both teeth and implants.

\section{ACKNOWLEDGMENTS}

The authors thank Dr. Stephen Meraw for his assistance in the preparation of this manuscript. This study was partially supported by the University of Michigan, Periodontal Graduate Student Research Fund.

\section{REFERENCES}

1. Ramfjord SP, Knowles JW, Nissle RR, Burgett FG, Shick RA. Results following three modalities of periodontal therapy. J Periodontol 1975;46:522-526.

2. Becker W, Becker BE, Ochsenbein C, et al. A longitudinal study comparing scaling, osseous surgery and modified Widman procedures. Results after one year. $J$ Periodontol 1988;59:351-365.

3. Kaldahl WB, Kalkwarf KL, Patil KD, Molvar MP, Dyer JK. Long-term evaluation of periodontal therapy: I. Response to 4 therapeutic modalities. J Periodontol 1996; 67:93-102.

4. Caton J, Nyman S. Histometric evaluation of periodontal surgery. I. The modified Widman flap procedure. J Clin Periodontol 1980;7:212-223.

5. Caton J, Nyman S, Zander H. Histometric evaluation of periodontal surgery. II. Connective tissue attachment levels after four regenerative procedures. J Clin Periodontol 1980;7:224-231.

6. Bowers GM, Chadroff B, Carnevale R, et al. Histologic evaluation of new attachment apparatus formation in humans. Part I. J Periodontol 1989;60:664-674.

7. Wilderman MN, Pennel BM, King K, Barron JM. Histogenesis of repair following osseous surgery. J Periodontol 1970;41:551-565.

8. The American Academy of Periodontology. Glossary of Periodontal Terms, 3rd ed. Chicago: The American Academy of Periodontology; 1992:42.

9. Garrett S. Periodontal regeneration around natural teeth. Ann Periodontol 1996;1:621-666.

10. Fritz ME. Implant therapy. II. Ann Periodontol 1996;1: 796-815.

11. Postlethwaite AE, Seyer JM, Kang AH. Chemotactic attraction of human fibroblasts to type I, II, and III collagens and collagen derived peptides. Proc Natl Acad Sci (USA) 1978;75:871-875.

12. Yaffe A, Ehrlich J, Shoshan S. Restoration of periodontal attachment employing enriched collagen solution in the dog. J Periodontol 1984;55:623-628.

13. Locci P, Calvitti M, Belcastro S, et al. Phenotype expression of gingival fibroblasts cultured on membranes used in guided tissue regeneration. J Periodontol 1997;68:857863.

14. Cooperman L, Michaeli D. The immunogenicity of 
injectable collagen I. A 1-year prospective study. J Am Acad Dermatol 1984;10:638-646.

15. Sableman E. Biology, Biotechnology and Biocompatibility of Collagen. Biocompatibility of Tissue Analogs, 1st ed. Boca Raton, FL: CRC Press, Inc; 1985:27.

16. Schlegel AK, Möhler H, Busch F, Mehl A. Preclinical and clinical studies of a collagen membrane (Bio-Gide). Biomaterials 1997;18:535-538.

17. Pitaru S, Tal H, Soldinger $M$, Noff $M$. Collagen membranes prevent apical migration of epithelium and support new connective tissue attachment during periodontal wound healing in dogs. J Periodont Res 1989;24:247253.

18. Quteish D, Singrao S, Dolby AE. Light and electron microscopic evaluation of biocompatability, resorption and penetration characteristics of human collagen graft material. J Clin Periodontol 1991;18:305-311.

19. Johns LP, Merritt K, Agarwal S, Ceravolo FJ. Immunogenicity of a bovine collagen membrane in guided tissue regeneration. J Dent Res 1992;71(Spec. Issue):298 (Abstr. 1538).

20. Wang H-L, O'Neal RB, Thomas CL, Shyr Y, MacNeil RL. Evaluation of an absorbable collagen membrane in treating class II furcation defects. J Periodontol 1994;65:10291036.

21. Unsal B, Kurtis B, Özcan G, Ozdemir A, Karaoz E. An investigation of resorption and tissue reaction after subcutaneous implantation of collagen based membrane materials in rats. J Marmara Univ Dent Fac 1997;2:609615.

22. Paul BF, Mellonig JT, Towle HJ, Gray JL. Use of a collagen barrier to enhance healing in human periodontal furcation defects. Int $J$ Periodontics Restorative Dent 1992;12:123-131.

23. Pitaru S, Tal H, Soldinger M, Grosskopf A, Noff M. Partial regeneration of periodontal tissues using collagen barriers. Initial observations in the canine. J Periodontol 1988;59:380-386.

24. Mayrand D, Grenier D. Detection of collagenase activity in oral bacteria. Can J Microbiol 1985;31:134-138.

25. Heath JK, Atkinson SJ, Hembry RM, Reynolds JJ, Meikle $M C$. Bacterial antigens induce collagenase and prostaglandin $E_{2}$ synthesis in human gingival fibroblasts through a primary effect on circulating mononuclear cells. Infect Immun 1987;55:2148-2154.

26. Selvig KA, Kersten BG, Chamberlain ADH, Wikesjö UME, Nilvéus RE. Regenerative surgery of intrabony periodontal defects using ePTFE barrier membranes: Scanning electron microscopic evaluation of retrieved membranes versus clinical healing. J Periodontol 1992;63: 974-978.

27. Nowzari H, MacDonald ES, Flynn J, London RM, Morrison JL, Slots J. The dynamics of microbial colonization of barrier membranes for guided tissue regeneration. J Periodontol 1996;67:694-702.

28. Chen Y-T, Wang H-L, Lopatin DE, O'Neal RB, MacNeil $\mathrm{RL}$. Bacterial adherence to guided tissue regeneration barrier membranes exposed to the oral environment. $J$ Periodontol 1997;68:172-179.

29. Minabe M, Kodama T, Kogou T, et al. Different crosslinked types of collagen implanted in rat palatal gingiva. $J$ Periodontol 1989;60:35-43.

30. Kodama T, Minabe $M$, Hori T, Watanabe $Y$. The effect of various concentrations of collagen barrier on periodontal wound healing. J Periodontol 1989;60:205-210.

31. Quteish D, Dolby AE. The use of irradiated-crosslinked human collagen membrane in guided tissue regenera- tion. J Clin Periodontol 1992;19:476-484.

32. Brunel G, Piantoni P, Elharar F, Benqué E, Marin P, Zahedi S. Regeneration of rat calvarial defects using a bioabsorbable membrane technique: Influence of collagen cross-linking. J Periodontol 1996;67:1342-1348.

33. Zahedi S, Legrand R, Brunel G, et al. Evaluation of a diphenylphosphorylazide-crosslinked collagen membrane for guided bone regeneration in mandibular defects in rats. J Periodontol 1998;69:1238-1246.

34. Numabe $Y$, Ito H, Hayashi H, Ryder MI, Kamoi K. Epithelial cell kinetics with atelocollagen membranes: A study in rats. J Periodontol 1993;64:706-712.

35. Crigger M, Bogle GC, Garrett S, Gantes BG. Repair following treatment of circumferential periodontal defects in dogs with collagen and expanded polytetrafluoroethylene barrier membranes. J Periodontol 1996;67:403413.

36. Speer DP, Chvapil M, Eskelson CD, Ulreich J. Biological effects of residual glutaraldehyde in glutaraldehydetanned collagen biomaterials. J Biomed Mater Res 1980;14:753-764.

37. Pitaru S, Noff M, Grosskopf A, Moses O, Tal H, Savion N. Heparan sulfate and fibronectin improve the capacity of collagen barriers to prevent apical migration of the junctional epithelium. J Periodontol 1991;62:598601.

38. Karring T, Nyman S, Lindhe J, Sirirat M. Potentials for root resorption during periodontal wound healing. J Clin Periodontol 1984;11:41-52.

39. Blumenthal NM. The use of collagen membranes to guide regeneration of new connective tissue attachment in dogs. J Periodontol 1988;59:830-836.

40. Cirelli JA, Marcantonio EJ, Adriana R, et al. Evaluation of anionic collagen membranes in the treatment of class II furcation lesions: An histometric analysis in dogs. Biomaterials 1997;18:1227-1234.

41. Tanner MG, Solt CW, Vuddhakanok S. An evaluation of new attachment formation using a microfibrillar collagen barrier. J Periodontol 1988;59:524-530.

42. Chung KM, Salkin LM, Stein MD, Freedman AL. Clinical evaluation of a biodegradable collagen membrane in guided tissue regeneration. J Periodontol 1990;61:732736.

43. al-Arrayed F, Adam S, Moran J, Dowell P. Clinical trial of cross-linked human type I collagen as a barrier material in surgical periodontal treatment. J Clin Periodontol 1995;22:371-379.

44. Mattson JS, McLey LL, Jabro MH. Treatment of intrabony defects with collagen membrane barriers. Case reports. J Periodontol 1995;66:635-645.

45. Benqué E, Zahedi S, Brocard D, Oscaby F, Justumus P, Brunel G. Guided tissue regeneration using a collagen membrane in chronic adult and rapidly progressive periodontitis patients in the treatment of 3-wall intrabony defects. J Clin Periodontol 1997;24:544-549.

46. Mattson JS, Gallagher SJ, Jabro MH. The use of 2 bioabsorbable barrier membranes in the treatment of interproximal intrabony periodontal defects. $J$ Periodontol 1999;70:510-517.

47. Tonetti MS, Pini-Prato G, Cortellini P. Periodontal regeneration of human intrabony defects. IV. Determinants of healing response. J Periodontol 1993;64:934-940.

48. Parodi R, Carusi G, Santarelli G, Nanni F, Pingitore R, Brunel G. Guided tissue regeneration employing a collagen membrane in a human periodontal bone defect: A histologic evaluation. Int J Periodontics Restorative Dent 1997; 17:283-291. 
49. Evans GH, Yukna RA, Cambre KM, Gardiner DL. Clinical regeneration with guided tissue barriers. Curr Opin Periodontol 1997;4:74-81.

50. Blumenthal NM, Steinberg J. The use of collagen membrane barriers in conjunction with combined demineralized bone-collagen gel implants in human infrabony defects. J Periodontol 1990;61:319-327.

51. Dowell P, al-Arrayed F, Adam S, Moran J. A comparative clinical study: The use of human type I collagen with and without the addition of metronidazole in the GTR method of treatment of periodontal disease. J Clin Periodontol 1995;22:543-549.

52. Chen CC, Wang H-L, Smith F, Glickman GN, Shyr Y, O'Neal RB. Evaluation of a collagen membrane with and without bone grafts in treating periodontal intrabony defects. J Periodontol 1995;66:838-847.

53. Benqué E, Zahedi S, Brocard D, Oscaby F, Justumus P, Brunel G. Combined collagen membrane and hydroxyapatite/collagen chondroitin-sulfate spacer placement in the treatment of 2-wall intrabony defects in chronic adult and rapidly progressive periodontitis patients. $J$ Clin Periodontol 1997;24:550-556.

54. Bower RC. Furcation morphology relative to periodontal treatment. Furcation root surface anatomy. J Periodontol 1979;50:366-374.

55. Masters DH, Hoskins SW. Projection of cervical enamel into molar furcations. J Periodontol 1964;35:49-53.

56. Everett FG, Jump EB, Holder TD, Williams GC. The intermediate bifurcational ridge: A study of the morphology of the bifurcation of the lower first molar. J Dent Res 1958;37:162-169.

57. Gutmanm JL. Prevalence, location, and patency of accessory canals in the furcation region of permanent molars. J Periodontol 1978;49:21-26.

58. Bower RC. Furcation morphology relative to periodontal treatment. Furcation entrance architecture. J Periodontol 1979;50:23-27.

59. Garrett S, Loos B, Chamberlain D, Egelberg J. Treatment of intraosseous periodontal defects with a combined adjunctive therapy of citric acid conditioning, bone grafting, and placement of collagenous membranes. J Clin Periodontol 1988;15:383-389.

60. Van Swol RL, Ellinger R, Pfeifer J, Barton NE, Blumenthal N. Collagen membrane barrier therapy to guide regeneration in class II furcations in humans. J Periodontol 1993;64:622-629.

61. Selvig KA, Nilvéus RE, Fitzmorris L, Kersten B, Khorsandi SS. Scanning electron microscopic observations of cell populations and bacterial contamination of membranes used for guided periodontal tissue regeneration in humans. J Periodontol 1990;61:515-520.

62. Blumenthal NM. A clinical comparison of collagen membranes with ePTFE membranes in the treatment of human mandibular buccal class II furcation defects. $J$ Periodontol 1993;64:925-933.

63. Black BS, Gher ME, Sandifer JB, Fucini SE, Richardson AC. Comparative study of collagen and expanded polytetrafluoroethylene membranes in the treatment of human class II furcation defects. J Periodontol 1994;65: 598-604.

64. Yukna CN, Yukna RA. Multi-center evaluation of bioabsorbable collagen membrane for guided tissue regeneration in human class II furcations. J Periodontol 1996;67: 650-657.

65. Tinti C, Vincenzi G, Cocchetto R. Guided tissue regeneration in mucogingival surgery. J Periodontol 1993;64: 1184-1191.
66. Scabbia A, Trombelli L. Long-term stability of the mucogingival complex following guided tissue regeneration in gingival recession defects. $J$ Clin Periodontol 1998;25:1041-1046.

67. Trombelli L, Scabbia A, Tatakis DN, Checchi L, Calura G. Resorbable barrier and envelope flap surgery in the treatment of human gingival recession defects. Case reports. J Clin Periodontol 1998;25:24-29.

68. Vincenzi G, De Chiesa A, Trisi P. Guided tissue regeneration using a resorbable membrane in gingival recession-type defects. A histologic case report in humans. Int J Periodontics Restorative Dent 1998;18:25-33.

69. Ricci G, Silvestri M, Tinti C, Rasperini G. A clinical/statistical comparison between the subpedicle connective tissue graft method and the guided tissue regeneration technique in root coverage. Int $J$ Periodontics Restorative Dent 1996;16:539-545.

70. Harris RJ. A comparative study of root coverage obtained with guided tissue regeneration utilizing a bioabsorbable membrane versus the connective tissue with partial-thickness double pedicle graft. J Periodontol 1997; 68:779-790.

71. Zucchelli G, Clauser C, De Sanctis M, Calandriello M. Mucogingival versus guided tissue regeneration procedures in the treatment of deep recession type defects. $J$ Periodontol 1998;69:138-145.

72. Jepsen K, Heinz B, Halben JH, Jepsen S. Treatment of gingival recession with titanium reinforced barrier membranes versus connective tissue grafts. J Periodontol 1998;69:383-391.

73. Parma-Benfenati S, Tinti C. Histologic evaluation of new attachment utilizing a titanium-reinforced barrier membrane in a mucogingival recession defect. A case report. $J$ Periodontol 1998;69:834-839.

74. Shieh A-T, Wang H-L, O'Neal R, Glickman GN, MacNeil RL. Development and clinical evaluation of a root coverage procedure using a collagen barrier membrane. $J$ Periodontol 1997;68:770-778.

75. Zahedi S, Bozon C, Brunel G. A 2-year clinical evaluation of a diphenylphosphorylazide-cross-linked collagen membrane for the treatment of buccal gingival recession. $J$ Periodontol 1998;69:975-981.

76. Özcan G, Kurtis B, Balos K. Combined use of root conditioning, fibrin-fibronectin system and a collagen membrane to treat a localized gingival recession: A 10-case report. J Marmara Univ Dent Fac 1997;2:588-598.

77. Wang H-L, MacNeil RL, Labadie M, Shyr Y, Bunyaratavej $P$. Clinical comparison of two techniques for treatment of gingival recession. J Dent Res 1999;78(Spec. Issue): 119(Abstr. 106).

78. Kimble K, Wang H-L, Eber R. Effect of additional bone grafts on GTR-based root coverage procedure. J Dent Res 2000;79(Spec. Issue):172(Abstr. 227).

79. Hämmerle CHF, Karring T. Guided bone regeneration at oral implant sites. Periodontol 2000 1998;17:151-175.

80. Iglhaut J, Aukhil I, Simpson DM, Johnston MC, Kock G. Progenitor cell kinetics during guided tissue regeneration in experimental periodontal wounds. J Periodont Res 1988;23:107-117.

81. Colangelo P, Piattelli A, Barrucci S, Trisi P, Formisano G, Caiazza S. Bone regeneration guided by resorbable collagen membranes in rabbits. A pilot study. Implant Dent 1993;2:101-105.

82. Sevor JJ, Meffert RM, Cassingham RJ. Regeneration of dehisced alveolar bone adjacent to endosseous dental implants utilizing a resorbable collagen membrane. Clinical and histologic results. Int J Periodontics Restorative 
Dent 1993;13:71-83.

83. Hürzeler MB, Kohal RJ, Naghshbandi J, et al. Evaluation of a new bioresorbable barrier to facilitate guided bone regeneration around exposed implant threads: An experimental study in the monkey. Int $J$ Oral Maxillofac Surg 1998;27:315-320.

84. Dongieux JW, Block MS, Morris G, Gardiner D, Dean K. The effect of different membranes on onlay bone graft success in the dog mandible. Oral Surg Oral Med Oral Pathol Oral Radiol Endod 1998;86:145-151.

85. Brunel G, Benqué E, Frederic E, et al. Guided bone regeneration for immediate nonsubmerged implant placement using bioabsorbable materials in beagle dogs. Clin Oral Implants Res 1998;9:303-312.

86. Zitzmann NU, Naef R, Schärer P. Resorbable versus nonresorbable membranes in combination with Bio-Oss for guided bone regeneration. Int $J$ Oral Maxillofac Implants 1997; 12:844-852.

87. Simion $M$, Baldoni $M$, Rossi P, Zaffe D. A comparative study of the effectiveness of e-PTFE membranes with and without early exposure during the healing period. Int $J$ Periodontics Restorative Dent 1994;14:167-180.

88. Parodi R, Carusi G, Santarelli G, Nanni F. Implant placement in large edentulous ridges expanded by GBR using a bioresorbable collagen membrane. Int $J$ Periodontics Restorative Dent 1998;18:267-275.

89. Wang H-L, MacNeil RL. Guided tissue regeneration: Absorbable barriers. Dent Clin North Am 1998;42:505522.
90. Choi SY, Nilvéus RE, Minutello RD, Zimmerman GJ, Wikesjö UME. Effect of a collagen matrix on healing in periodontal fenestration defects in dogs. $J$ Periodontol 1993;64:878-882.

Send reprint requests to: Dr. Hom-Lay Wang, Department of Periodontics/Prevention/Geriatrics, University of Michigan School of Dentistry, 1011 North University Ave., Ann Arbor, MI 48109-1078. Fax: 734/936-0374; e-mail: homlay@umich.edu

Accepted for publication July 7, 2000. 